\title{
Vertical-flow paper SERS system for therapeutic drug monitoring of flucytosine in serum
}

\author{
Adam G. Berger, Stephen M. Restaino, and Ian M. White*
}

Fischell Department of Bioengineering, University of Maryland, College Park, MD 20742. *ianwhite@umd.edu

Keywords. Surface enhanced Raman Spectroscopy, SERS, Paper sensors, Therapeutic drug monitoring, flucytosine, point-of-care, vertical flow.

Abstract. A number of life-saving drugs require therapeutic drug monitoring (TDM) for safe and effective use. Currently, however, TDM is performed using sophisticated analytical techniques relegated to central labs, increasing the cost per test and time to answer. Here, using a novel vertical flow membrane system with inkjet-printed surface enhanced Raman sensors, along with a portable spectrometer, we demonstrate a low cost and easy to use device to quantify levels of flucytosine, an antifungal that requires TDM for effective patient care, from undiluted human serum. To our knowledge, this work represents the first report of a passive vertical flow sample cleanup method with surface enhanced Raman detection. We first investigated and optimized the parameters of the vertical flow system for the detection of flucytosine in spiked serum samples. Then, using an optimized vertical-flow system utilizing nitrocellulose membranes and a paper SERS sensor, we achieved detection of down to $10 \mu \mathrm{g} \mathrm{mL} \mathrm{m}^{-1}$ flucytosine in undiluted serum, with quantitative detection across the entire therapeutic range. This system reduces the 
assay time to about 15 minutes, far quicker than the current gold standards. We anticipate that this novel system will enable near-patient therapeutic drug monitoring, leading to the safe and effective administration of a number of life-saving drugs. Furthermore, it will spawn the development of SERS detection systems capable of separating target analytes from real-world biological matrices. 


\section{Introduction}

Recommendations to monitor the real-time physiological load of therapeutic drugs in patients, referred to as therapeutic drug monitoring (TDM), have been increasing with the move towards precision medicine [1-3]. In particular, TDM may be necessary for therapeutics with a narrow window of efficacy or for therapeutics with a high variability in renal elimination among patients. Classes of drugs that typically warrant TDM include antimicrobials [4-8], chemotherapeutics $[9,10]$, and anti-psychotics [11,12], among others.

One of the most documented cases in which TDM can dramatically improve patient outcomes is that of the antifungal drug flucytosine (5-fluorocytosine - 5FC). Flucytosine is primarily used to treat infections with Candida species and Cryptococcus neoformans [13,14], and is critical for treatment of Cryptococcal Meningitis [4,15]. In fungal cells, cytosine deaminase converts the 5FC form into the functional 5-fluorouracil (5FU) form, which causes RNA miscoding and inhibits DNA synthesis, thus interfering with protein synthesis $[5,15,16]$. While it is effective in the appropriate blood concentration, toxic side effects can occur in high doses, including bone marrow suppression, hepatotoxicity, and gastrointestinal toxicity [16-18]. One study suggests that up to $40 \%$ of patients receiving 5FC develop hepatotoxicity [16], and in a study of nearly 200 patients, $91 \%$ of patients showed bone marrow suppression after four weeks of treatment [18]. The commonly accepted threshold for toxicity is a serum concentration of $100 \mu \mathrm{g} \mathrm{mL}^{-1}$ [4,5,15-19]. Meanwhile, the minimum inhibitory concentration (MIC) for fungal infections is on the order of $10 \mu \mathrm{g} \mathrm{mL}^{-1}$ [15], and commonly recommended doses for a range of fungal infections are often between $20 \mu \mathrm{g} \mathrm{mL}^{-1}$ and $80 \mu \mathrm{g} \mathrm{mL} L^{-1}$, depending on the targeted species $[13,14,16,17,20]$. $5 \mathrm{FC}$ dosage is further complicated by the fact that there is high variability in renal elimination of 5FC [13]. To illustrate this, a study of over 200 patients 
showed that only about $20 \%$ of patients had $5 \mathrm{FC}$ levels within the expected range, while about $40 \%$ had levels below the target dose and about $40 \%$ had levels above the target dose, of which about $10 \%$ were toxic [14]. Moreover, for Cryptococcal Meningoencephalitis, 5FC is often administered as a cocktail with amphotericin B (AmB) [19], which can cause renal impairment, thus adding to the variability of 5FC load in patients. As a result, the need for TDM of flucytosine is widely recognized $[4,13,15-17]$.

Current methods for TDM do not meet the need for a quick, inexpensive, portable, multiplex-capable, near real-time, and sensitive determination of serum drug concentration. Available methods include microbiological assays, high-performance liquid chromatography (HPLC), and liquid chromatography-mass spectrometry (LC-MS) [4,13,21]. Microbiological assays require manual steps, culturing, and are vulnerable to interference when combination antifungal therapy is used $[4,13]$. Standard analytical chemistry techniques (e.g., LC/MS) are more sensitive and allow rapid quantification even when combination antifungal therapy is used; however, they are expensive and require complex equipment operated by skilled technicians. Thus, samples must be transported to a central laboratory facility for analysis and queued $[4,13]$. It is clear that a near-patient solution for flucytosine TDM is necessary to improve patient outcomes.

Surface enhanced Raman spectroscopy (SERS) has been investigated as an alternative to traditional analytical chemistry techniques for chemical and biomolecular analysis. SERS is based upon Raman scattering, the inelastic scattering of light unique to molecular vibrations inherent in the molecule. Though Raman scattering is a relatively rare event, noble metal nanostructures provide plasmonic enhancement to the intensity of Raman scattering [22-24]. While in most cases SERS cannot compete with HPLC/MS for sensitivity and specificity in a 
central lab setting, SERS may be capable of meeting applications away from the central lab, including analysis in the field and in near-patient settings, aided by the emergence of highperforming portable and handheld Raman spectrometers. To date, SERS has been examined for TDM of the chemotherapeutics methotrexate and 5-fluorouracil [25-27], aminoglycosides [28], the anti-epileptic valproic acid [29], and a host of IV drugs [30]; however, reports of SERS for TDM from applicable bodily fluids, such as serum or saliva are still lacking, as are pragmatic demonstrations applicable to detection in the field.

While miniaturized spectrometers can lead to portability, SERS can only be applied for TDM in near-patient settings if sample processing is simplified, as serum, plasma, or whole blood samples present challenges for direct SERS analysis. Components within serum foul the plasmonic surface and thus inhibit direct interactions with the targeted therapeutic. New sample preparation methods integrated with SERS sensors must be developed. In particular, proposed techniques must not require labor- or equipment-intensive intervention steps to meet the needs of portability and ease-of-use.

Traditional SERS sensors were not designed with these constraints in mind. Historically, SERS sensors have been fabricated onto rigid substrates using relatively sophisticated synthesis techniques, leading to high chip costs or limited mass production capabilities. Alongside the cost of production, rigid substrates provide only basic analytic functionality and therefore require offchip equipment and manual steps for sample acquisition and processing. To address these limitations, our group developed inkjet-printed paper-based SERS sensors as low cost and easy to use sensors for chemical and biomolecular analysis [31-34]. While paper based SERS sensors were first reported 30 years ago $[35,36]$, it has only emerged as a realistic solution for chemical 


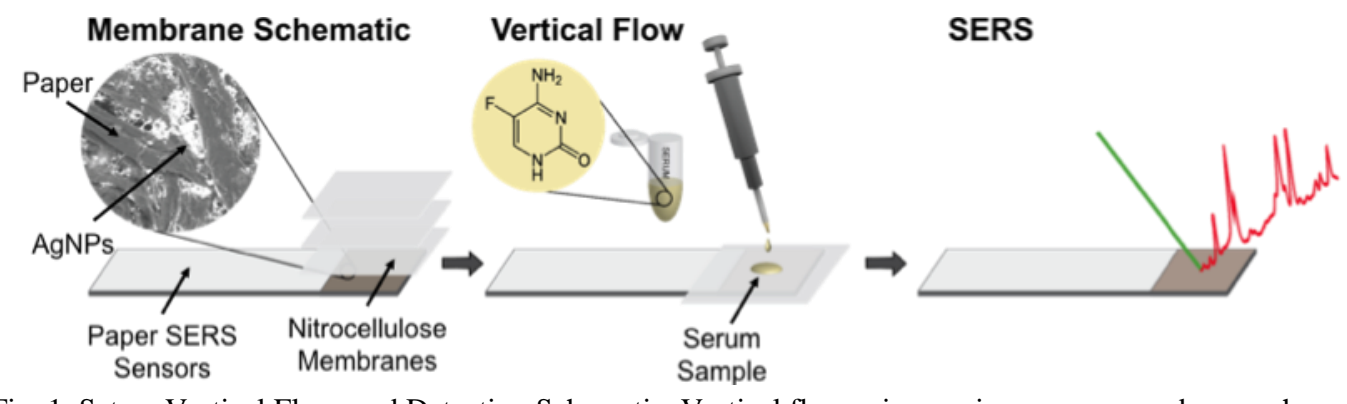

Fig. 1. Setup, Vertical Flow, and Detection Schematic. Vertical flow using a microporous membrane scheme separates the flucytosine from the serum. SEM of the inkjet-printed paper AgNPs on cellulose paper is shown. The SERS signal is read from the paper SERS sensors.

analytics within the last 5 years, starting with reports of high-performing substrates fabricated with inkjet printing [34], screen printing [37], and soaking [38].

The cost of printed sensors on paper substrates is clearly an advantage for near-patient applications, but equally important are the sample acquisition and cleanup capabilities. We demonstrated the inherent capabilities of paper SERS sensors to collect samples from surfaces via swabbing or from liquid via dipping [32]. We also reported integrated sample cleanup through detection of melamine in infant formula by using chromatography on a PVDF membrane that had been decorated with silver nanoparticles via inkjet fabrication [33].

In this work, we demonstrate a new scheme to monitor flucytosine in undiluted serum, which can be broadly applied in many other TDM applications. Two previous reports have demonstrated SERS for TDM applications in diluted serum. One report measured a chemotherapeutic in serum [26], though the limit of detection prevented quantification across the therapeutic range, likely because of variability introduced from the lack of sample cleanup. Another report demonstrated detection of tobramycin, an aminoglycoside antibiotic, in serum with a 1000-fold dilution, but the detection at this low dilution was only possible with the aid of a Raman reporter [28]. Furthermore, the need for a dilution likely inhibits near-patient applications because of the need for precise intervention steps. Our solution for TDM with 
undiluted serum samples is illustrated in Fig. 1. A sample droplet is applied to a membrane selected to trap serum components while transmitting the therapeutic. Beneath the filtering membrane is an inkjet-fabricated paper SERS sensor, which wicks the sample into the sensor from the membrane. The potential fouling agents are trapped in the nitrocellulose, while the 5FC passes through the membrane and adsorbs onto the nanoparticles. The sensor is easily interrogated with a portable Raman spectrometer.

Using this vertical flow design, we report the measurement of 5FC in undiluted serum across the entire therapeutic window. Although vertical flow assays have been shown in the past, to the best of our knowledge none have been used for SERS or for pump-free separation of undiluted serum [39-42]. We first show that utilizing nitrocellulose as the filtering membrane results in the highest discrimination, as other materials may not bind protein as well or may prevent the passage of 5FC. We then optimize the number of nitrocellulose membranes in the vertical flow stack, the sample volume, and the amount of time allowed for vertical flow. In addition, we investigate the impact of diluting the serum sample and confirm that our system performs best without diluting the sample. Finally, we demonstrate quantitative detection of 5FC spiked into human serum from $150 \mu \mathrm{g} \mathrm{mL}^{-1}$ to $10 \mu \mathrm{g} \mathrm{mL}$, allowing analysis of concentrations beyond the thresholds for toxicity $\left(100 \mu \mathrm{g} \mathrm{mL}^{-1}\right)$ and ineffectiveness $\left(20 \mu \mathrm{g} \mathrm{mL} \mathrm{m}^{-1}\right)$.

\section{Materials and Methods}

\subsection{Materials}

Whatman Grade 1 chromatography paper was purchased as a reel from GE Healthcare (Pittsburgh, PA). Nitrocellulose membranes ( $0.45 \mu \mathrm{m}$ pores) were obtained as a reel from BioRad (Hercules, CA). Durapore Hydrophilic polyvinylidene fluoride (PVDF) Membranes (0.1 
$\mu \mathrm{m}$ pores, $90 \mathrm{~mm}$ diameter) and Durapore Hydrophobic PVDF Membranes (0.22 $\mu \mathrm{m}$ pores, 13 mm diameter) were purchased from Millipore (Darmstadt, Germany). Polycarbonate membrane filters $(5 \mu \mathrm{m}$ pores, $25 \times 80 \mathrm{~mm})$ and nylon membrane filters $(5 \mu \mathrm{m}$ pores, $25 \mathrm{~mm}$ diameter $)$ were obtained from Sterlitech (Kent, WA). Trisodium citrate dehydrate, silver nitrate, 1,2-Di(4Pyridyl)ethylene (BPE), and flucytosine secondary pharmaceutical standard were purchased from Sigma-Aldrich (St. Louis, MO). Propylene glycol was obtained from SAFC (St. Louis, MO). Hydrochloric acid ( $\mathrm{HCl})$ was purchased from BDH Chemicals (West Chester, PA). AB Human Serum was obtained from Invitrogen (Brown Deer, WI) and stored at $-20^{\circ} \mathrm{C}$ until use. All reagents were handled according to their respective safety data sheet.

\subsection{AgNP Synthesis and Ink Formation}

Citrate capped silver nanoparticles (AgNPs) were synthesized based on a modified citrate reduction, as originally proposed by Lee and Meisel [43]. Briefly, $400 \mathrm{~mL}$ of ultrapure water is brought to a boil, at which point $72 \mathrm{mg} \mathrm{AgNO}_{3}$ and $80 \mathrm{mg}$ trisodium citrate are added. The solution is boiled for 10 minutes with stirring set so that the vortex touches the bottom of the flask. The AgNPs can be stored at $4^{\circ} \mathrm{C}$ until use. A printable ink is formed from the AgNP colloid. Briefly, the AgNP colloid is centrifuged at $10000 \mathrm{rpm}$ and $4{ }^{\circ} \mathrm{C}$ for 20 minutes in $50 \mathrm{~mL}$ conical tubes. The supernatant is removed, leaving approximately $1 \%$ of the original volume. The remaining concentrated colloid is combined with propylene glycol (70:30 AgNPs to propylene glycol) and vortexed well.

\subsection{Paper Sensor Fabrication}


The AgNP ink is printed onto Whatman Grade 1 cellulose chromatography paper using a Deskjet 1010 inkjet printer (HP, Palo Alto, CA). The tops of the ink cartridges are opened, emptied, and cleaned; $350 \mu \mathrm{L}$ of the AgNP ink is then pipetted into the cartridge. An arrayed pattern of the sensors is drawn in MATLAB (Mathworks, Natick, MA) and printed onto the paper 15 times, waiting 10 minutes every 5 cycles to allow the sensor spots to dry. Sensors are

dried under vacuum for at least 30 minutes and stored in a desiccator until use. In this paper, all sensors were used within 24 hours of printing for consistency. Sensors are cut to $0.5 \times 0.5 \mathrm{~cm}$ with a $1.5 \times 0.5 \mathrm{~cm}$ handle (Fig. 2 A). Sensors are imaged with a S-3400 Variable Pressure SEM (Hitachi, Schaumburg, IL) with backscatter detection at $15 \mathrm{kV}$ and $100 \mathrm{~Pa}$ of pressure.

\subsection{Vertical Flow Separation from Serum}

For measurements without vertical flow (i.e., direct detection), $2 \mu \mathrm{L}$ samples are dropped on the printed AgNPs using a pipette. For separation from serum, a vertical flow setup is used, as shown in Fig. 2B. The sensor is positioned with the sensor face up on a glass slide. The microporous membranes are placed on top of the nanoparticle detection zone. To align the vertical flow components, we illuminate the underside of the membranes to visualize the sensor. Sample droplets are placed on top of the uppermost microporous membrane directly over the nanoparticle region of the underlying SERS sensor. Another glass slide is placed directly on top of the membranes and the entire setup is clamped with a pony-style spring clamp to maintain contact between membranes and the sensor.

Unless otherwise stated, all optimizations were performed with $10 \mu \mathrm{L} 100 \mu \mathrm{g} \mathrm{mL} \mathrm{m}^{-1}$ 5FC in $100 \%$ serum. In experiments aimed at identifying the best membrane material, one membrane and a 5 minute vertical flow assay time were used. To determine simultaneously the optimal 


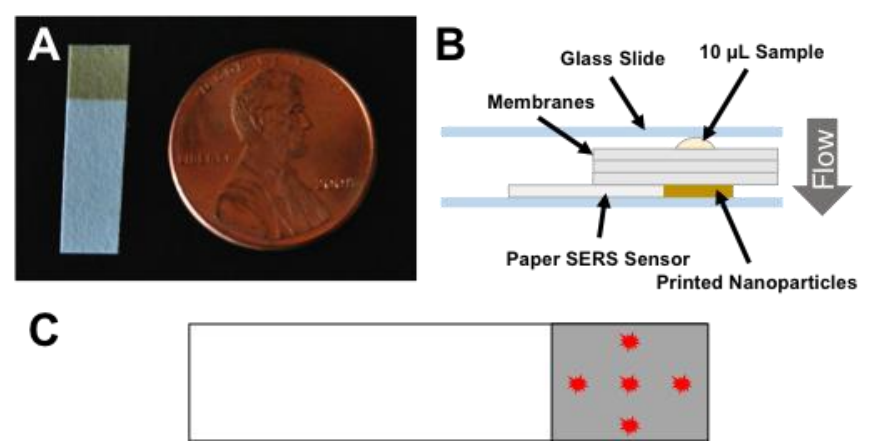

Fig. 2. Methods. A) The paper SERS sensors are printed and cut to be approximately $0.5 \times 2 \mathrm{~cm}$ with a $0.5 \times 0.5 \mathrm{~cm}$ detection zone. B) Membranes are stacked to allow vertical flow and separate the 5FC from the serum. C) Spectra are acquired at the location of the red stars.

number of filtering membranes and sample volume, we used a 5 minute vertical flow assay time while varying the sample volume and the number of membranes. We then varied the assay time from 2 to 20 minutes, keeping an assay configuration with three membranes. Finally, we optimized the serum dilution from 100 to 20 percent while using three membranes and an assay time of 10 minutes. These same conditions were used to test the sensor and membrane geometries.

\subsection{SERS Measurements}

SERS measurements are performed using a portable Raman system consisting of a $785 \mathrm{~nm}$ diode laser (Innovative Photonic Solutions, Monmouth Junction, NJ), QE65000 Raman spectrometer (Ocean Optics, Dunedin, FL), and fiber optic probe (Integrated Photonic Solutions, Monmouth Junction, NJ). Laser power is set to approximately $15 \mathrm{~mW}$. Each spectrum is collected using a 1 second CCD exposure for 3 accumulations (averaged). Spectra are averaged across 5 spots, shown in Fig. 2C. To minimize variability, the spots were collected in the same locations on each sensor. 


\subsection{Spectral Analysis}

The spectra were analyzed using in-house MATLAB code. Each spectrum was backgroundsubtracted using a sextic fitting and code adapted from the literature [44]. Our code determines the maximum number of counts between the pixels that are associated with Raman shifts from

783.5 to $788.7 \mathrm{~cm}^{-1}$. The number of counts is averaged for each sensor. The average counts of the three sensors and the associated standard deviation across the three sensors are reported. The least-squares regression line and Langmuir fits are calculated as appropriate to model the data. The parameters for the Langmuir fit are chosen to maximize the $\mathrm{R}^{2}$ value.

\section{Results and Discussion}

\subsection{Characterization of $5 \mathrm{FC}$ with SERS}

To identify the characteristic Raman peaks of 5FC, we began by recording a conventional Raman spectrum of 5FC powder. Figure $3 \mathrm{~A}$ highlights the key peaks of $5 \mathrm{FC}$ in the measured spectrum. Of note, the most intense peak occurs at $784 \mathrm{~cm}^{-1}$, which is likely attributed to the ring breathing mode [45]. This peak was thus selected to quantify the intensity of the spectra of 5FC for all future quantification experiments. The intensity peak height is referred to as $I_{784}$.

To record a SERS spectrum and compare it to the Raman spectrum, 5FC is prepared in aqueous solution and dropped onto a paper SERS sensors. Figure 3B presents both spectra. Many of the key peaks are the same between the two representative spectra, although they may be slightly shifted or vary in intensity. Some of the peaks in the Raman spectrum are not present in the SERS spectrum, as is typical for SERS. 

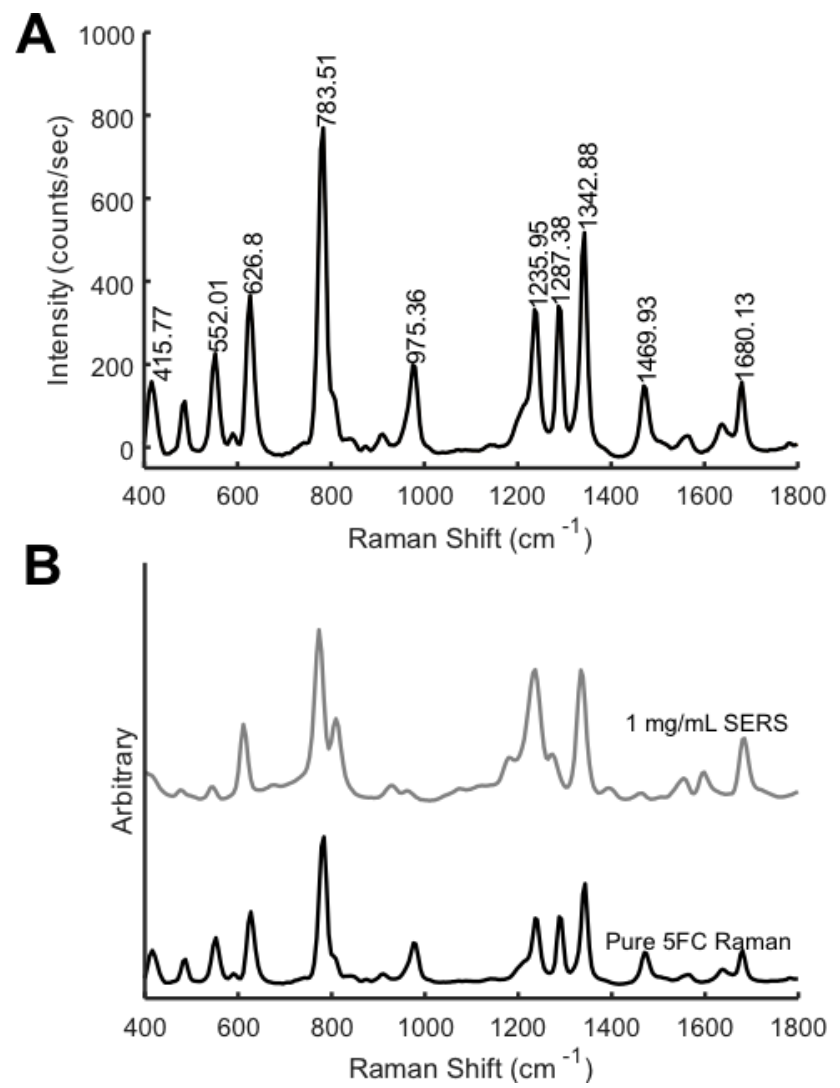

Fig. 3. 5FC Raman spectrum and sample SERS spectrum. A) The average Raman of the 5FC powder is collected on an aluminum foilcovered glass slide. A prominent peak occurs at about $784 \mathrm{~cm}^{-1}$. B) The average normalized Raman spectrum of 5FC is compared to the average normalized SERS of $1 \mu \mathrm{g} \mathrm{mL}^{-1} 5 \mathrm{FC}$ in water on the paper SERS substrate. Many peaks overlap between the Raman and SERS spectra, although they may be slightly shifted. The spectra are intensity normalized to better highlight similarity. Offset is artificial.

\subsection{Detection of 5FC with Paper SERS}

To assess the capability of paper SERS sensors to measure 5FC at physiologically relevant concentrations, 5FC was diluted in DI water in decreasing concentrations. Sample droplets of $2 \mu \mathrm{L}$ were dropped on the paper SERS surfaces and interrogated with the Raman spectrometer. Figure 4A shows the measured spectra for a representative selection of the concentrations tested $\left(1000,75,25,10,5,2.5\right.$, and $\left.0 \mu \mathrm{gL}^{-1} 5 \mathrm{FC}\right)$. The spectra are artificially offset to highlight their differences. Fig. 4B shows a zoomed in version of the spectra for 5FC at concentrations of 5, 2.5, 1, 0.5 , and $0 \mu \mathrm{g} \mathrm{mL}{ }^{-1} 5 \mathrm{FC}$. Even at $0.5 \mu \mathrm{gL}^{-1}$, the $\mathrm{I}_{784}$ signal is visible. The relevant peak 

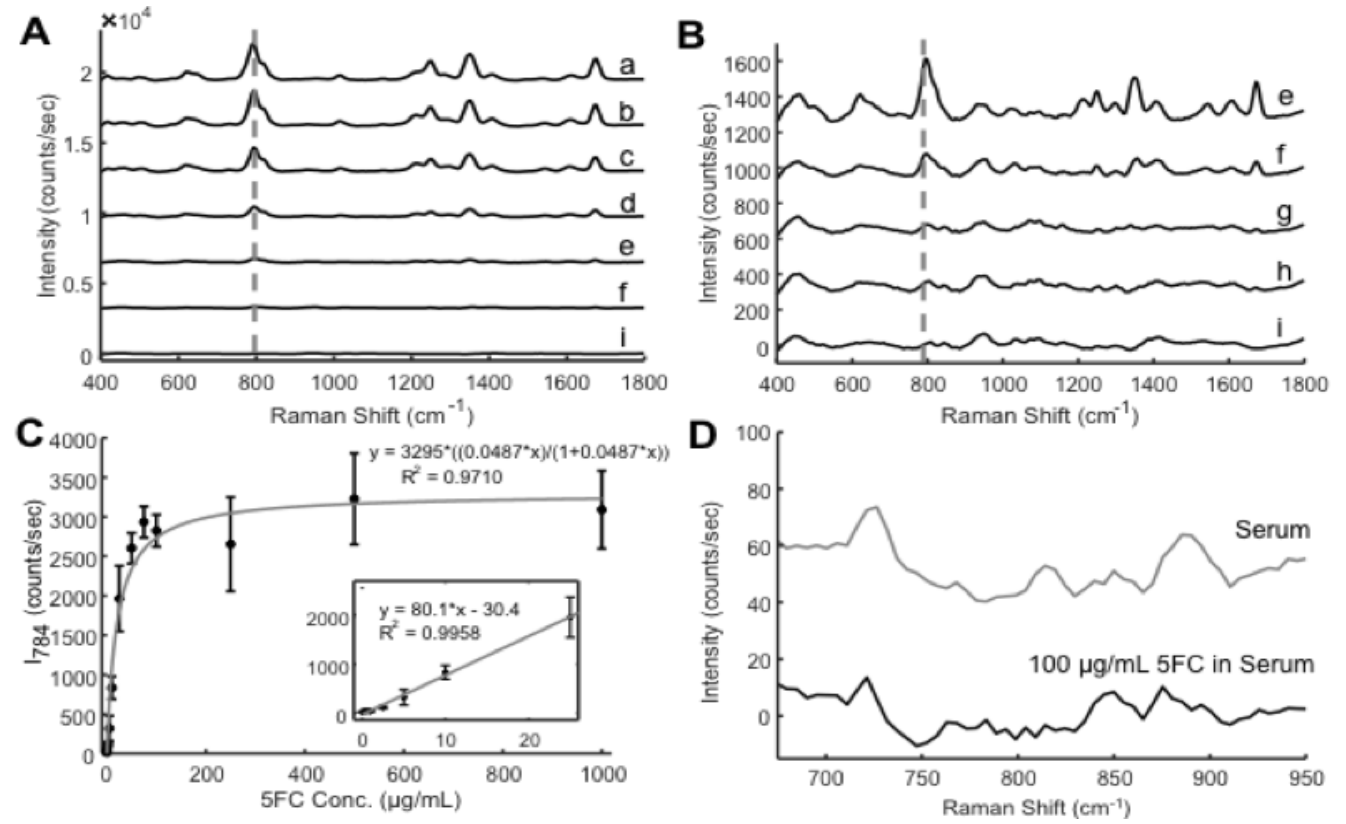

Fig. 4. Detection performance for $5 \mathrm{FC}$ in water. A) $2 \mu \mathrm{L}$ of $5 \mathrm{FC}$ in water at the following concentrations (in $\mu \mathrm{g} \mathrm{mL}^{-1}$ ) was dropped onto the sensor and spectra were collected: (a) 1000, (b) 75, (c) 25, (d) 10, (e) 5, (f) 2.5, (g) 1 , (h) 0.5 , (i) 0 . Offset is artificial. B) An enhanced view of the spectra for lower concentrations from Fig. 4A is shown. C) The intensity at $780 \mathrm{~cm}^{-1}$ was used to generate a calibration curve. A Langmuir isotherm was fit to the data with an $\mathrm{R}^{2}$ of 0.971 . Inset shows the signal at lower concentrations. Error bars show the standard deviation. D) $2 \mu \mathrm{L}$ of $100 \%$ serum and $100 \mu \mathrm{g} \mathrm{mL}^{-1} 5 \mathrm{FC}$ in $100 \%$ serum were dropped on the paper SERS sensor surface and spectra were collected. No appreciable 5FC signal was observed in the average spectra, with any small peaks likely attributed to the serum. Offset is artificial.

heights increased linearly for concentrations of $0-25 \mu \mathrm{g} \mathrm{mL} L^{-1}$ (Inset). At about $50 \mu \mathrm{g} \mathrm{mL}^{-1}$, the $\mathrm{I}_{784}$ signal begins to saturate, as expected for a sensor with finite surface area. In Fig. 4C, the full sample range $\left(0-1000 \mu \mathrm{g} \mathrm{mL}^{-1}\right)$ was fit to a Langmuir isotherm with the coefficient of determination $\left(\mathrm{R}^{2}=0.971\right)$ indicating strong predictive potential up to $1000 \mu \mathrm{g} \mathrm{mL} \mathrm{L}^{-1}$. Similarly,

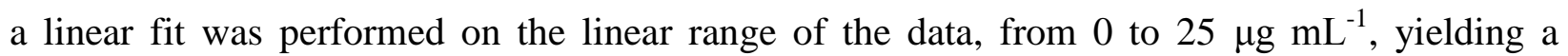
coefficient of determination of 0.996 , again indicating strong predictive potential in that range.

The variability presented in Fig. 4C by the error bars represents the standard deviation across three sensors. The inter-sensor variability is highlighted in Fig. S1 (Supplemental Material), where the standard deviation across five points (shown in Fig. 2C) on three different sensors is investigated. While we collect and average data across five points for each sensor to 
account for the variation, rastering (a feature included in some new handheld spectrometers) will improve and automate this process [46].

Referring again to the inter-sensor standard deviation shown in Fig. 4C, the resolution based on 3 times the standard deviation of the signal at $0 \mu \mathrm{g} \mathrm{mL} L^{-1}$ is 4.2 counts $\mathrm{sec}^{-1}$. Within the linear range, the sensitivity, given by the slope of the linear fit, is 80.1 counts $\sec ^{-1}$ per $\mu \mathrm{g} \mathrm{mL} L^{-1}$. Thus,

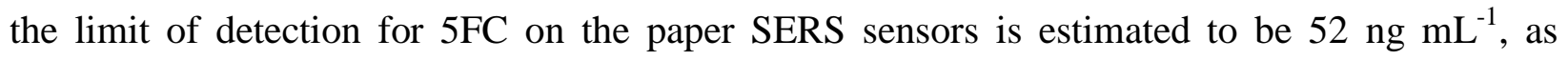
determined by dividing the resolution by the sensitivity.

To determine the impact of serum on the capability to detect 5FC, spiked samples with 100 $\mu \mathrm{g} \mathrm{mL} \mathrm{L}^{-1} 5 \mathrm{FC}$ were spotted directly onto the sensor surface, and SERS spectra were acquired. Fig. 4D demonstrates the inability to detect $5 \mathrm{FC}$ in serum without any separation, as no significant peaks appear around $784 \mathrm{~cm}^{-1}$. It is readily apparent that the serum is inhibiting interactions between the analyte and the noble metal surfaces.

\subsection{Vertical-flow separation with paper SERS}

We have previously shown that a lateral flow arrangement and an appropriately selected mobile phase could be used to detect melamine from infant formula [33]. However, the insolubility of 5FC in volatile solvents that typically serve as a mobile phase inhibited this ability, and thus the lateral flow separation and detection scheme is ineffective. In order to separate the 5FC from serum, we used a passive vertical flow scheme that leverages both the wicking properties and protein retention properties of microporous membranes to selectively deliver the 5FC to the sensor surface. Vertical flow allows for a similar protein binding area as lateral flow, but decreases the distance for mass transfer of the separated 5FC. Although vertical flow assays have been shown in the past, to the best of our knowledge this is the first 


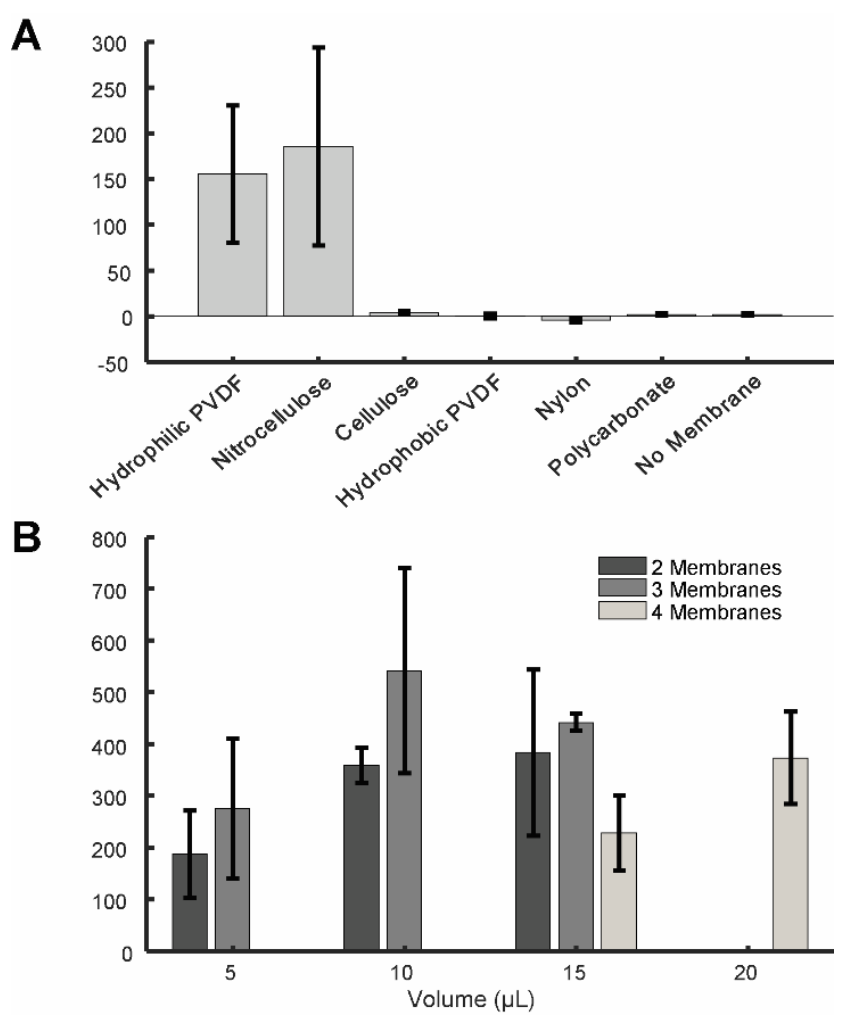

Fig. 5. Optimization of vertical flow scheme for 5FC detection from serum. $100 \mu \mathrm{g} \mathrm{mL}-15 \mathrm{FC}$ in serum was tested using the lateral flow scheme and varying the A) microporous membrane materials and B) the number of nitrocellulose membranes and sample volume. Error bars show the standard deviation.

demonstration incorporating SERS as well as pump-free separation of drugs from serum [3942]. To optimize this vertical flow scheme, a number of parameters were varied, including the membrane type, the number of membranes, the sample volume, the assay time, the serum dilution, and the size of the microporous membranes. Each optimization parameter was tested on three sensors, and the inter-sensor standard deviation was calculated.

Microporous membrane materials were assessed first. As shown in Fig. 5A, both the hydrophilic PVDF and nitrocellulose membranes allowed a good vertical flow of the 5FC while retaining the serum, as the $\mathrm{I}_{784}$ peak was visible (in contrast to Fig. 4D). It is likely that cellulose, nylon, and polycarbonate membranes failed to retain a majority of the serum components, while the hydrophobic version of PVDF prevents liquid flow. Nitrocellulose was ultimately selected as the membrane for this vertical flow scheme. Nitrocellulose is commonly 
used in molecular biology due to its wettability and its known affinity for proteins [47]. Note in Fig. 5A that hydrophilic PVDF may also be an option, as it also shares these same properties with nitrocellulose.

After selecting nitrocellulose as the filtering membrane for this assay, the number of nitrocellulose membranes in the vertical flow stack was varied along with the sample volume to test any interplay between these two variables. As one would expect, increasing the sample volume requires additional filtering membranes to retain the serum components, though if the sample is too small to penetrate the selected number of membranes, the signal intensity is penalized. Figure 5B shows the results of an assessment across these two parameters. The results suggest that three membranes (with a $10 \mu \mathrm{L}$ sample volume) appear to provide a balance between filtering fouling agents and enabling mass transfer of the 5FC via vertical flow to the sensor surface.

We also investigated the dependence of the measured SERS signal intensity on the allowed time for vertical flow. Fig. S2 (Supplemental Material) shows that the $\mathrm{I}_{784}$ signal due to 5FC SERS increases proportionally from 2 to 5 minutes (following sample addition). Beyond 5 minutes, the $\mathrm{I}_{784}$ signal continues to increase, though with a diminishing slope. After 20 minutes, 5FC continues to transfer to the sensor, and it appears as though complete saturation has not been reached. However, we expect that a short time to read will be a priority in clinical near-patient settings, so we limited future experiments to 10 minutes. Nonetheless, it appears that the detection limit in this vertical flow arrangement could be modestly improved if necessary by allowing the assay to run for an extended time, such as 30 minutes.

We then investigated the dependence of diluting the sample. We hypothesized that because our system removes the serum components, the optimal solution would be to avoid diluting the 
sample, as this delivers the maximum amount of drug to the system while avoiding preparation steps. A solution of $100 \mu \mathrm{g} \mathrm{mL}^{-1} 5 \mathrm{FC}$ in $100 \%$ serum was diluted with ultrapure water to $80 \%$, $60 \%, 40 \%$, and $20 \%$ serum. The measured $\mathrm{I}_{784}$ intensity for the various samples is presented in Fig. S3 (Supplemental Material). Interestingly, the signal was about the same for 100\%, 80\%, $60 \%$, and $40 \%$ serum, suggesting that the lower serum concentration may have aided in the detection of $5 \mathrm{FC}$, though this effect is balanced by the decreasing concentration of $5 \mathrm{FC}$ in the sample. For $20 \%$ serum, the signal dropped by about half compared to $40 \%$ serum, suggesting that diluting the serum below $40 \%$ has no benefit, while the decreasing $5 \mathrm{FC}$ leads to a reduced signal. Given these results, all subsequent tests used 100\% serum, as the elimination of hands-on steps leads to a quicker and easier assay.

In addition to the optimizations described above, we assessed the dependence of the assay's detection performance on the geometry of the sensor as well as the geometry of the filter. We hypothesized that a larger filter may wick 5FC away from the sensor, thus leading to a lower signal. We also hypothesized that extended wicking pads fanning away from the SERS sensor may more effectively draw sample into the sensor, thus increasing the SERS signal. However, as shown in Figures S4 and S5 (Supplemental Material), detection of 5FC in serum appears to be relatively independent of both the sensor and filter geometry. 


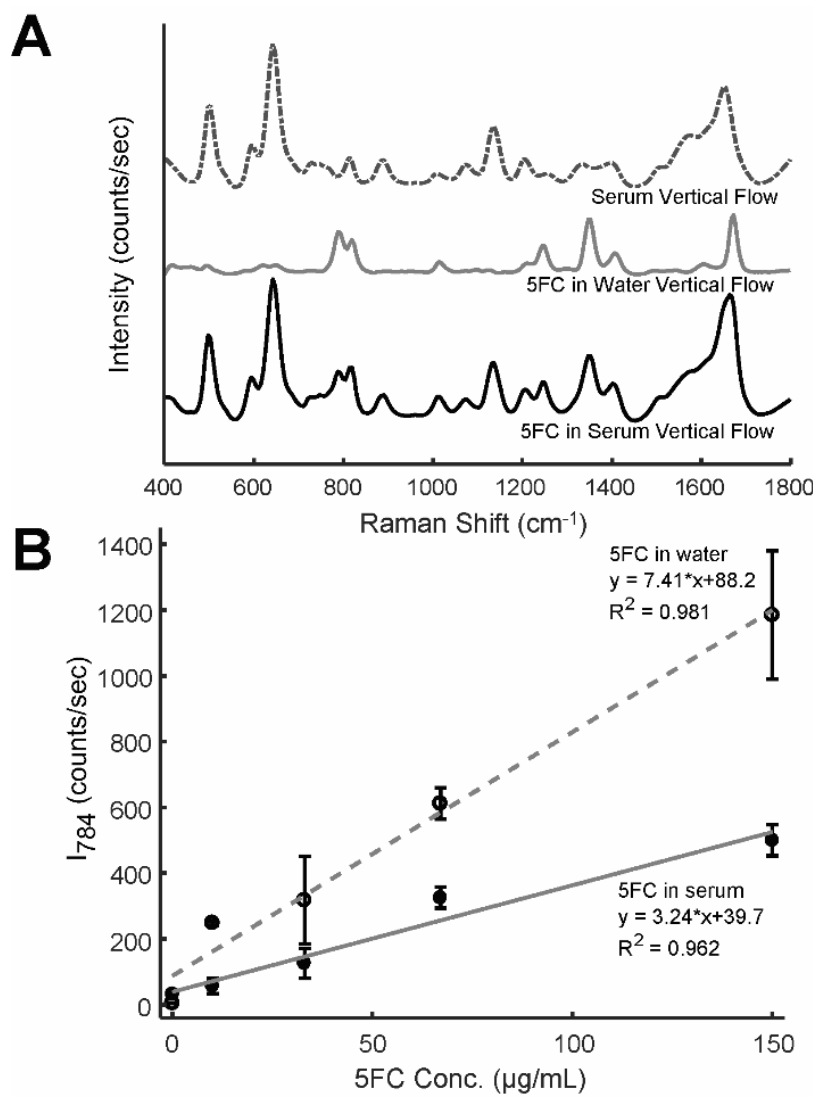

Fig. 6. Calibration curve of 5FC using vertical flow. A) The vertical flow scheme is tested with serum alone and 5FC in both water and serum. Average spectra are shown with arbitrary scaling and artificial offset. Additional peaks appear in the flow from serum, but the characteristic 5FC peaks are visible. B) Spectra were taken of 5FC in serum or 5FC in water at varying concentrations after vertical flow separation. Results are shown and a linear fit is calculated for both 5FC in serum and 5FC in water with good agreement.

\subsection{Detection limit of $5 F C$ in Serum}

Using the selected assay scheme (3 nitrocellulose membranes, $10 \mu \mathrm{L}$ sample volume, 10 min assay time, $100 \%$ serum), the detection performance of 5FC in serum was investigated. The vertical flow assay was tested with $5 \mathrm{FC}$ in both serum and water, as shown in Fig. 6A. The characteristic 5FC peak at $784 \mathrm{~cm}^{-1}$ was readily observed in vertical flow from both water and serum, as are other Raman peaks related to 5FC. Of note, other Raman peaks appear in the signal recorded from the serum sample, especially around $500 \mathrm{~cm}^{-1}, 640 \mathrm{~cm}^{-1}, 1140 \mathrm{~cm}^{-1}$, and a broad peak around $1660 \mathrm{~cm}^{-1}$ that is blue-shifted from the 5FC peak around that region. These 
peaks align with those in the serum sample with no 5FC, thus leading us to suspect that these peaks are due to some of the components of serum that passed through the microporous membranes. Although the $784 \mathrm{~cm}^{-1} 5 \mathrm{FC}$ peak is close to overlapping with some of the serum peaks, it is still more distinguishable from the background as compared to other peaks and thus was used to quantify the 5FC concentration. Chemometrics with multivariate analyses could possibly further distinguish the signal from the noise, as has been demonstrated $[26,48]$. Nonetheless, it is clear from the 5FC Raman signal that sensor fouling was effectively diminished, contrary to the case without the membranes (Fig. 4D).

Figure 6B presents the calibration curves for the detection of 5FC with SERS sensors in the vertical flow separation scheme (spectra are shown in Fig. S6, Supplemental Material). Data using vertical flow from both serum and water are shown, and a linear regression is fit to the data. 5FC recovery from serum using SERS detection is shown for five data points throughout the therapeutic range of $5 \mathrm{FC}$, including detection of down to $10 \mu \mathrm{g} \mathrm{mL}^{-1} 5 \mathrm{FC}$. The signal intensity for given concentrations in comparison with Fig. 4 indicates that only about $6 \%$ of the 5FC reaches the sensor when the three membranes are used; despite this, the ability of the vertical filter stack to sequester inhibitors from the serum dramatically improves the capability of the sensor to measure 5FC in serum. To estimate the detection limit of the vertical flow system, we identify the concentration along the line fit that corresponds to three standard deviations above the background, which yields $12.1 \mu \mathrm{g} \mathrm{mL}^{-1}$, in good agreement with the visible detection of $10 \mu \mathrm{gL}^{-1}$ 5FC in Fig. 6B and supplemental Fig. S6. This limit of detection, along with the linear range of detection out to at least $150 \mu \mathrm{g} \mathrm{mL}^{-1}$, demonstrates the ability of our paper SERS sensor in the vertical flow scheme to measure $5 \mathrm{FC}$ in serum across the clinically relevant therapeutic range. 
As expected, the capabilities of the system to detect 5FC in water were significantly better than in serum (Fig. 6B). The detection limit, defined as the concentration along the line fit that corresponds to three standard deviations above the background, is $222 \mathrm{ng} \mathrm{mL}{ }^{-1}$. This limit of detection for $5 \mathrm{FC}$ in water using the vertical flow scheme is not far from the detection limit for 5FC applied directly onto the sensor, further validating the ability of our vertical flow biosensing scheme as a way to block serum components from fouling the sensor surface while still allowing the 5FC to flow through.

\section{Conclusion}

A critical need exists for near-patient therapeutic drug monitoring to improve patient outcomes. Currently TDM can be performed with conventional analytical chemistry techniques, but this limits the analysis to central labs, leading to expensive tests with delayed results. Complicating the transition to a near-patient implementation is the need for sample cleanup, as detection of therapeutics in serum samples can be obfuscated by the complex serum matrix. SERS sensors on paper or membrane substrates, which can be fabricated through low-cost and high-throughput inkjet printing, present a potential solution as paper and membranes have inherent capabilities to acquire and cleanup samples.

Here we report the first demonstration of TDM with inkjet-printed paper SERS sensors and a passive vertical flow membrane system. We leverage a vertical flow system with nitrocellulose membranes to filter serum components and a cellulose SERS sensor to wick the remaining sample volume. We successfully demonstrate that the vertical flow format removes the serum components that inhibit detection while enabling the targeted drug, 5FC, to reach the sensor efficiently. We optimized the design of the vertical flow configuration and investigated the dependence of sample volume and dilution. In addition, we showed that the vertical flow 
system only moderately decreases performance for detection of $5 \mathrm{FC}$, while enabling detection of 5FC in $100 \%$ serum across the therapeutic range. Our tested linear range $\left(10 \mu \mathrm{g} \mathrm{mL} \mathrm{L}^{-1}\right.$ to $150 \mu \mathrm{g}$

$\mathrm{mL}^{-1}$ ) provides our method with a model capable of identifying serum concentrations of 5FC below the therapeutic dose $\left(20 \mu \mathrm{gL}^{-1}\right)$ and above the threshold for toxicity $\left(100 \mu \mathrm{g} \mathrm{mL}^{-1}\right)$, potentially allowing for monitoring and maintenance of this life-saving therapeutic.

A critical next step is to include the capability to filter cells from whole blood. We anticipate that this can be implemented with an additional membrane at the top of the vertical flow system, as others have demonstrated blood cell removal with membranes [49,50]. Furthermore, broadening the evaluation of the system to other drugs is an important direction. Of note, while TDM of 5FC only requires the detection of the drug and not the metabolite due to the low concentration of the latter in the blood, many other applications may require the drug and metabolite to be distinguished, which is an important consideration for the use of SERS. Completion of these next steps will lead to pragmatic near-patient bleed-to-read TDM assays from a droplet of blood.

\section{Acknowledgments}

This work was supported by the National Science Foundation (ECCS1149850), the Maryland Industrial Partnerships program, and Diagnostic anSERS, Inc. Additional support for this research was provided by a grant to the University of Maryland from the Howard Hughes Medical Institute Undergraduate Science Education Program. The authors acknowledge the support of the Maryland NanoCenter, its FabLab, and Mr. Jonathan Hummel for help with SEM (The NanoCenter is supported in part by the NSF as a MRSEC Shared Experimental Facility). The authors acknowledge Mr. John Goertz for fruitful discussions on biosensor development. 
Ms. Megan Dang and Mr. Adriel Sumathipala assisted with printing SERS sensors. Ms. Imaly

Nanayakkara assisted with photography.

\section{References}

[1] S. Jang, Z. Yan, J. Lazor, Therapeutic drug monitoring: A patient management tool for precision medicine, Clin. Pharmacol. Ther. 99 (2016) 148-150. doi:10.1002/cpt.298.

[2] A. Dasgupta, Therapeutic Drug Monitoring: Newer Drugs and Biomarkers, Academic Press, 2012.

[3] K.S. McKeating, A. Aubé, J.-F. Masson, Biosensors and nanobiosensors for therapeutic drug and response monitoring, Analyst. 141 (2016) 429-449. doi:10.1039/C5AN01861G.

[4] H.R. Ashbee, R.A. Barnes, E.M. Johnson, M.D. Richardson, R. Gorton, W.W. Hope, Therapeutic drug monitoring (TDM) of antifungal agents: guidelines from the British Society for Medical Mycology, J. Antimicrob. Chemother. 69 (2014) 1162-1176. doi:10.1093/jac/dkt508.

[5] E.J. Begg, M.L. Barclay, C.M.J. Kirkpatrick, The therapeutic monitoring of antimicrobial agents, Br. J. Clin. Pharmacol. 52 (2001) 35-43. doi:10.1111/j.1365-2125.2001.00377.x.

[6] F. Follath, M. Wenk, S. Vozeh, Plasma concentration monitoring of aminoglycosides, J. Antimicrob. Chemother. 8 (1981) 37-43. doi:10.1093/jac/8.suppl_A.37.

[7] W.W. Hope, E.M. Billaud, J. Lestner, D.W. Denning, Therapeutic drug monitoring for triazoles, Curr. Opin. Infect. Dis. 21 (2008) 580-586. doi:10.1097/QCO.0b013e3283184611.

[8] M. Rybak, B. Lomaestro, J.C. Rotschafer, R. Moellering, W. Craig, M. Billeter, J.R. Dalovisio, D.P. Levine, Therapeutic monitoring of vancomycin in adult patients: A consensus review of the American Society of Health-System Pharmacists, the Infectious Diseases Society of America, and the Society of Infectious Diseases Pharmacists, Am. J. Health Syst. Pharm. 66 (2009) 82-98. doi:10.2146/ajhp080434.

[9] L. Alnaim, Therapeutic drug monitoring of cancer chemotherapy, J. Oncol. Pharm. Pract. 13 (2007) 207-221. doi:10.1177/1078155207081133.

[10] M.E. de Jonge, A.D.R. Huitema, J.H.M. Schellens, S. Rodenhuis, J.H. Beijnen, Individualised Cancer Chemotherapy: Strategies and Performance of Prospective Studies on Therapeutic Drug Monitoring with Dose Adaptation, Clin. Pharmacokinet. 44 (2012) 147-173. doi:10.2165/00003088-200544020-00002.

[11] R. Eilers, Therapeutic Drug Monitoring for the Treatment of Psychiatric Disorders, Clin. Pharmacokinet. 29 (2012) 442-450. doi:10.2165/00003088-199529060-00005.

[12] A.Y. Khan, S.H. Preskorn, Examining Concentration-Dependent Toxicity of Clozapine: Role of Therapeutic Drug Monitoring, J. Psychiatr. Pract. 11 (2005) 289-301.

[13] D. Andes, A. Pascual, O. Marchetti, Antifungal Therapeutic Drug Monitoring: Established and Emerging Indications, Antimicrob. Agents Chemother. 53 (2009) 24-34. doi:10.1128/AAC.00705-08. 
[14] A.C. Pasqualotto, S.J. Howard, C.B. Moore, D.W. Denning, Flucytosine therapeutic monitoring: 15 years experience from the UK, J. Antimicrob. Chemother. 59 (2007) $791-$ 793. doi:10.1093/jac/dk1550.

[15] A. Loyse, F. Dromer, J. Day, O. Lortholary, T.S. Harrison, Flucytosine and cryptococcosis: time to urgently address the worldwide accessibility of a 50-year-old antifungal, J. Antimicrob. Chemother. 68 (2013) 2435-2444. doi:10.1093/jac/dkt221.

[16] A. Vermes, H.-J. Guchelaar, J. Dankert, Flucytosine: a review of its pharmacology, clinical indications, pharmacokinetics, toxicity and drug interactions, J. Antimicrob. Chemother. 46 (2000) 171-179. doi:10.1093/jac/46.2.171.

[17] M.M. Chau, D.C.M. Kong, S.J. van Hal, K. Urbancic, J.A. Trubiano, M. Cassumbhoy, J. Wilkes, C.M. Cooper, J.A. Roberts, D.J.E. Marriott, L.J. Worth, Consensus guidelines for optimising antifungal drug delivery and monitoring to avoid toxicity and improve outcomes in patients with haematological malignancy, 2014, Intern. Med. J. 44 (2014) 1364-1388. doi:10.1111/imj.12600.

[18] A.M. Stamm, R.B. Diasio, W.E. Dismukes, S. Shadomy, G.A. Cloud, C.A. Bowles, G.H. Karam, A. Espinel-Ingroff, Toxicity of amphotericin B plus flucytosine in 194 patients with cryptococcal meningitis, Am. J. Med. 83 (1987) 236-242. doi:10.1016/00029343(87)90691-7.

[19] J.R. Perfect, W.E. Dismukes, F. Dromer, D.L. Goldman, J.R. Graybill, R.J. Hamill, T.S. Harrison, R.A. Larsen, O. Lortholary, M.-H. Nguyen, P.G. Pappas, W.G. Powderly, N. Singh, J.D. Sobel, T.C. Sorrell, Clinical Practice Guidelines for the Management of Cryptococcal Disease: 2010 Update by the Infectious Diseases Society of America, Clin. Infect. Dis. 50 (2010) 291-322. doi:10.1086/649858.

[20] M. Schulz, S. Iwersen-Bergmann, H. Andresen, A. Schmoldt, Therapeutic and toxic blood concentrations of nearly 1,000 drugs and other xenobiotics, Crit. Care. 16 (2012) R136. doi:10.1186/cc11441.

[21] J.W.C. Alffenaar, A.M.A. Wessels, K. van Hateren, B. Greijdanus, J.G.W. Kosterink, D.R.A. Uges, Method for therapeutic drug monitoring of azole antifungal drugs in human serum using LC/MS/MS, J. Chromatogr. B. 878 (2010) 39-44. doi:10.1016/j.jchromb.2009.11.017.

[22] M. Moskovits, Persistent misconceptions regarding SERS, Phys. Chem. Chem. Phys. 15 (2013) 5301-5311. doi:10.1039/C2CP44030J.

[23] M. Moskovits, Surface-enhanced Raman spectroscopy: a brief retrospective, J. Raman Spectrosc. 36 (2005) 485-496. doi:10.1002/jrs.1362.

[24] P.L. Stiles, J.A. Dieringer, N.C. Shah, R.P.V. Duyne, Surface-Enhanced Raman Spectroscopy, Annu. Rev. Anal. Chem. 1 (2008) 601-626. doi:10.1146/annurev.anchem.1.031207.112814.

[25] S. Farquharson, C. Shende, F.E. Inscore, P. Maksymiuk, A. Gift, Analysis of 5-fluorouracil in saliva using surface-enhanced Raman spectroscopy, J. Raman Spectrosc. 36 (2005) 208212. doi:10.1002/jrs.1277.

[26] S. Fornasaro, S.D. Marta, M. Rabusin, A. Bonifacio, V. Sergo, Toward SERS-based pointof-care approaches for therapeutic drug monitoring: the case of methotrexate, Faraday Discuss. 187 (2016) 485-499. doi:10.1039/C5FD00173K.

[27] I.J. Hidi, A. Mühlig, M. Jahn, F. Liebold, D. Cialla, K. Weber, J. Popp, LOC-SERS: towards point-of-care diagnostic of methotrexate, Anal. Methods. 6 (2014) 3943-3947. doi:10.1039/C3AY42240B. 
[28] K.S. McKeating, M. Couture, M.-P. Dinel, S. Garneau-Tsodikova, J.-F. Masson, High throughput LSPR and SERS analysis of aminoglycoside antibiotics, Analyst. 141 (2016) 5120-5126. doi:10.1039/C6AN00540C.

[29] M. Yokoyama, T. Nishimura, K. Yamada, M. Kido, Y. Ohno, Y. Sakurai, D. Mita, Y. Yamaguchi, E. Tamiya, T. Nagakura, Feasibility study of paper-based surface enhanced Raman spectroscopy of tear fluids for onsite therapeutic drug monitoring, in: 2014 World Automation Congress (WAC), 2014: pp. 474-477. doi:10.1109/WAC.2014.6936005.

[30] H.-Y. Wu, B.T. Cunningham, Point-of-care detection and real-time monitoring of intravenously delivered drugs via tubing with an integrated SERS sensor, Nanoscale. 6 (2014) 5162-5171. doi:10.1039/C4NR00027G.

[31] E.P. Hoppmann, W.W. Yu, I.M. White, Highly sensitive and flexible inkjet printed SERS sensors on paper, Methods. 63 (2013) 219-224. doi:10.1016/j.ymeth.2013.07.010.

[32] W.W. Yu, I.M. White, Inkjet-printed paper-based SERS dipsticks and swabs for trace chemical detection, Analyst. 138 (2013) 1020-1025. doi:10.1039/c2an36116g.

[33] W.W. Yu, I.M. White, Chromatographic separation and detection of target analytes from complex samples using inkjet printed SERS substrates, Analyst. 138 (2013) 3679-3686. doi:10.1039/C3AN00673E.

[34] W.W. Yu, I.M. White, Inkjet Printed Surface Enhanced Raman Spectroscopy Array on Cellulose Paper, Anal. Chem. 82 (2010) 9626-9630. doi:10.1021/ac102475k.

[35] C.D. Tran, In situ identification of paper chromatogram spots by surface enhanced Raman scattering, J. Chromatogr. 292 (1984) 432-438.

[36] C.D. Tran, Subnanogram detection of dyes on filter paper by surface-enhanced Raman scattering spectrometry, Anal. Chem. 56 (1984) 824-826. doi:10.1021/ac00268a057.

[37] L.-L. Qu, D.-W. Li, J.-Q. Xue, W.-L. Zhai, J.S. Fossey, Y.-T. Long, Batch fabrication of disposable screen printed SERS arrays, Lab Chip. 12 (2012) 876-881. doi:10.1039/C2LC20926H.

[38] C.H. Lee, L. Tian, S. Singamaneni, Paper-Based SERS Swab for Rapid Trace Detection on Real-World Surfaces, ACS Appl. Mater. Interfaces. 2 (2010) 3429-3435. doi:10.1021/am1009875.

[39] T. Chinnasamy, L.I. Segerink, M. Nystrand, J. Gantelius, H.A. Svahn, Point-of-Care Vertical Flow Allergen Microarray Assay: Proof of Concept, Clin. Chem. 60 (2014) 12091216. doi:10.1373/clinchem.2014.223230.

[40] Y.K. Oh, H.-A. Joung, S. Kim, M.-G. Kim, Vertical flow immunoassay (VFA) biosensor for a rapid one-step immunoassay, Lab Chip. 13 (2013) 768-772. doi:10.1039/C2LC41016H.

[41] P. Reuterswärd, J. Gantelius, H.A. Svahn, An 8 minute colorimetric paper-based reverse phase vertical flow serum microarray for screening of hyper IgE syndrome, Analyst. 140 (2015) 7327-7334. doi:10.1039/C5AN01013F.

[42] S. Wang, C. Zhang, J. Wang, Y. Zhang, Development of colloidal gold-based flow-through and lateral-flow immunoassays for the rapid detection of the insecticide carbaryl, Anal. Chim. Acta. 546 (2005) 161-166. doi:10.1016/j.aca.2005.04.088.

[43] P.C. Lee, D. Meisel, Adsorption and surface-enhanced Raman of dyes on silver and gold sols, J. Phys. Chem. 86 (1982) 3391-3395. doi:10.1021/j100214a025.

[44] V. Mazet, C. Carteret, D. Brie, J. Idier, B. Humbert, Background removal from spectra by designing and minimising a non-quadratic cost function, Chemom. Intell. Lab. Syst. 76 (2005) 121-133. doi:10.1016/j.chemolab.2004.10.003. 
[45] J. Florián, V. Baumruk, J. Leszczyński, IR and Raman Spectra, Tautomeric Stabilities, and Scaled Quantum Mechanical Force Fields of Protonated Cytosine, J. Phys. Chem. 100 (1996) 5578-5589. doi:10.1021/jp953284w.

[46] P.A. Mosier-Boss, M.D. Putnam, The Evaluation of Two Commercially Available, Portable Raman Systems, Anal. Chem. Insights. 8 (2013) 83-97. doi:10.4137/ACI.S11870.

[47] H. Towbin, T. Staehelin, J. Gordon, Electrophoretic transfer of proteins from polyacrylamide gels to nitrocellulose sheets: procedure and some applications, Proc. Natl. Acad. Sci. U. S. A. 76 (1979) 4350-4354.

[48] C.R. Yonzon, C.L. Haynes, X. Zhang, Walsh Joseph T., R.P. Van Duyne, A Glucose Biosensor Based on Surface-Enhanced Raman Scattering: Improved Partition Layer, Temporal Stability, Reversibility, and Resistance to Serum Protein Interference, Anal. Chem. 76 (2004) 78-85. doi:10.1021/ac035134k.

[49] O.B. Ayyub, A.M. Behrens, B.T. Heligman, M.E. Natoli, J.J. Ayoub, G. Cunningham, M. Summar, P. Kofinas, Simple and inexpensive quantification of ammonia in whole blood, Mol. Genet. Metab. 115 (2015) 95-100. doi:10.1016/j.ymgme.2015.04.004.

[50] L. Lin, J.T. Guthrie, Preparation and characterisation of novel, blood-plasma-separation membranes for use in biosensors, J. Memb. Sci. 173 (2000) 73-85. doi:10.1016/S03767388(00)00350-1. 


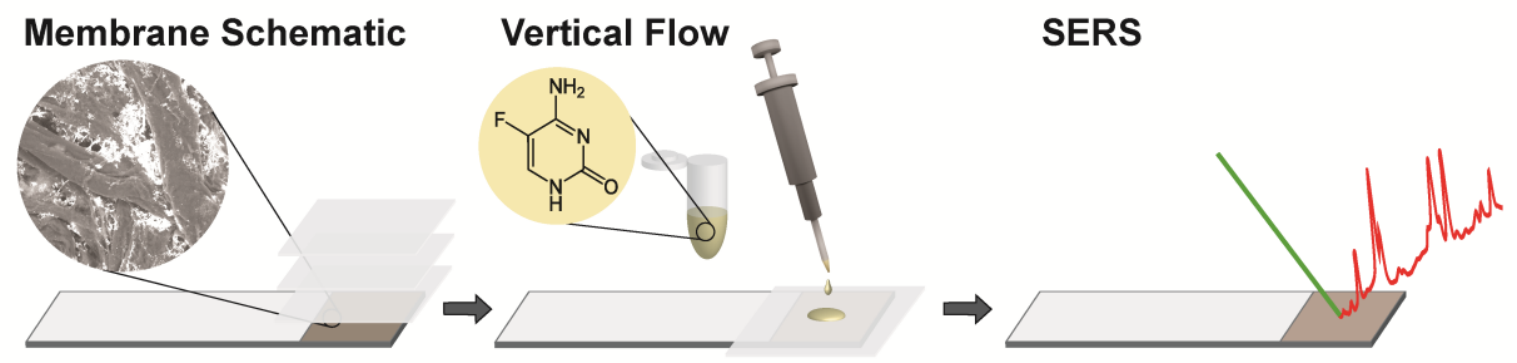

